\title{
A ghost of dioecy past and the legacy of sexual dimorphism: low siring
}

\section{2}

3

4

5

6 $7 \quad$ Switzerland

8 7UH4

1

\section{success of hermaphrodites after the breakdown of dioecy}

Luis Santos del Blanco ${ }^{1,2,3}$, Eleri Tudor ${ }^{4,5}$ and John R. Pannell ${ }^{1,6}$

${ }^{1}$ Department of Ecology and Evolution, Biophore Building, University of Lausanne, 1015 Lausanne,

${ }^{2}$ Current address: Sustainable Forest Management Research Institute University of Valladolid - INIA, Avda. Madrid 34071 Palencia, Spain

${ }^{3}$ Current address: Biotecnología Forestal Aplicada, Calle Curtidores 17, 34004 Palencia, Spain

${ }^{4}$ Department of Plant Sciences, University of Oxford, Oxford OX1 3RB, UK

${ }^{5}$ Current address: Crop Genetics Department, John Innes Centre, Norwich Research Park, Norwich NR4

${ }^{6}$ Author for correspondence: john.pannell@unil.ch, tel. +41-21-692-41-70

Statement of authorship: LS and JRP conceived the study. LS and EL carried out the experiments. LS conducted all analysis and made the figures. JRP wrote the manuscript with help from LS. All authors commented and approved the manuscript for submission.

Running title: Sexual dimorphism and the breakdown of dioecy

Key words: dioecy, hermaphroditism, monoecy, sexual dimorphism, inflorescence architecture,

Mercurialis annua, gain curves, sex allocation 


\section{Abstract}

26 Evolutionary transitions from dioecy to hermaphroditism must overcome the inertia of sexual

27 dimorphism because modified males or females will express the opposite sexual function for which their

28 phenotypes have been optimized. We tested this prediction by comparing the siring success of female-

29 derived hermaphrodites of the plant Mercurialis annua with males and hermaphrodites that present a

30 male-like inflorescence. We found that pollen dispersed by female-derived hermaphrodites was about a

31 third poorer at siring outcross offspring than that from hermaphrodites with male-like inflorescences,

32 illustrating the notion that a 'ghost of dioecy past' compromises the fitness of derived hermaphrodites

33 in outcrossing populations. We conclude that whereas dioecy might evolve from hermaphroditism by

34 conferring upon individuals certain benefits of sexual specialization, reversals from dioecy to

35 hermaphroditism must often be limited to situations in which outcrossing cannot be maintained and

36 inbreeding is favored. Our study provides novel empirical support for evolutionary models for the

37 breakdown of dioecy. 
39 Dioecy is found in about $6 \%$ of species, but in about half of all families of flowering plants (Renner \& Ricklefs 1995; Weiblen et al. 2000; Renner 2014). This distribution might suggest that dioecy is an evolutionary dead end or 'failure' (Westergaard 1958; Bull \& Charnov 1985; Heilbuth 2000), such that

42 lineages with separate sexes diversify less and are more prone to extinction than their hermaphroditic counterparts. However, the dead-end hypothesis has been challenged by analysis suggesting that the evolution of dioecy might actually increase lineage diversification, and that the scattered phylogenetic distribution of dioecious species might be explained by frequent reversions from dioecy to functional hermaphroditism, i.e., to a state in which plants have either bisexual flowers or flowers of both sexes

47 ('monoecy') (Kafer et al. 2014; Käfer \& Mousset 2014; Sabath et al. 2016; Kafer et al. 2017).

49 Evidence for reversions from dioecy to hermaphroditism has been accumulating for some time. Phylogenetic analysis of clades in which both dioecy and hermaphroditism occur indicates that dioecy

51 was probably not derived from, but was ancestral to, hermaphroditism (Goldberg et al. 2017; Kafer et al.

52 2017), including, e.g., the large and successful Cucurbitaceae family, in which it was lost several times

53 (Volz \& Renner 2008; Schaefer \& Renner 2010), and in the genera Bursera (Becerra \& Venable 1999),

54 Garcinia (Sweeney 2008), Gallium (Soza \& Olmstead 2010) and Dodonaea (Harrington \& Gadek 2010).

55 Within genera, Goldberg et al. (2017) found that transitions towards combined sexes were no less common than those towards separate sexes. In many of these reversions, dioecy evolved from

57 monoecy, not hermaphroditism with bisexual flowers, suggesting that the association between dioecy

58 and monoecy (Renner \& Ricklefs 1995) may be explained not only by the evolution of dioecy from

59 hermaphroditism via monoecy, but also by the breakdown of dioecy to monoecy.

60

61 The breakdown of dioecy presumably involves the selection of individuals with 'leaky' sex expression, 
62 i.e., males producing occasional fruits and seeds, or females producing occasional flowers with

63 functional stamens and pollen. Leaky sex expression is common in dioecious populations (reviewed in

64 Ehlers \& Bataillon 2007; Cossard et al. 2018), and has been invoked in models for the breakdown of

65 dioecy in both plants (Crossman \& Charlesworth 2014) and animals (Pannell 2008). Lloyd (1975a)

66 suggested that dioecy had broken down in Leptinella as a result of selection of leaky males following

67 colonization. Similarly, monoecy in the Mercurialis annua species complex probably evolved from

ancestral dioecy (Krahenbuhl et al. 2002; Obbard et al. 2006) under selection for reproductive assurance

69 in metapopulations with frequent colonization (Pannell 2001).

70

71 Although the breakdown of dioecy clearly involves changes in sex expression and sex allocation, the

72 relative fitness of males, females and invading hermaphrodites must also depend on the extent to which

73 the dioecious population is sexually dimorphic for non-reproductive traits. Sexual dimorphism is almost

74 ubiquitous in dioecious plants, with males and females differing in morphological, physiological,

75 defense, life-history, resource acquisition and inflorescence traits (Darwin 1871; Geber et al. 1999;

76 Fairbairn et al. 2007; Moore \& Pannell 2011; Barrett \& Hough 2013). The evolution of sexual

77 dimorphism likely allows males and females in dioecious populations to express phenotypes that

78 enhance siring success and seed production, respectively, but that might compromise these functions if

79 expressed in the other sex (Lande 1980; Cox \& Calsbeek 2009). Yet reversion to hermaphroditism must

80 bring about just this sort of compromise, because modified males or females will express their newly

81 acquired function in the context of a phenotype optimized for the opposite sex. The evolution of sexual

82 dimorphism should constrain the breakdown of dioecy in a way that goes beyond a simple sex-allocation

83 trade-off.

84

85 The likely constraints of sexual dimorphism on the breakdown of dioecy are well illustrated in wind- 
pollinated species, in which male and female inflorescences are often quite different (Lloyd \& Webb

1977; Whitehead 1983; Weberling 1992; Galonka et al. 2005; Friedman \& Barrett 2009b; Harris \&

Pannell 2010; Harder \& Prusinkiewicz 2013). Male (or staminate) flowers of wind-pollinated species are

typically held on flexible stalks or 'peduncles', which facilitate pollen liberation from anthers and pollen dispersal by wind (reviewed in Harder \& Prusinkiewicz 2013). In trees, these structures often hang from the branches. In herbs, they are typically held above the plant canopy. In both situations, pollen is liberated when turbulent gusts shake male flowers or anthers (Urzay et al. 2009). In contrast, female (or pistillate) flowers are typically held on more rigid inflorescences, and pollen is picked up by stigmas as they impact its surface, or from non-viscous eddies around the flower (but see Cresswell et al. 2010). Whereas the two sexual functions of bisexual hermaphrodite flowers will often interfere with one another in wind-pollinated plants, compromising fitness (Friedman \& Barrett 2009b; Harder \&

97 Prusinkiewicz 2013), the inflorescences of plants with separate male or female flowers (in dioecious or monoecious species) may be optimized for each sex separately (Friedman \& Barrett 2008), e.g., male flowers of Zea mays are born on flexible tassels at the shoot apex, whereas female flowers develop in the leaf axils (Aylor et al. 2003).

101

The male and female inflorescences of the plant Mercurialis annua L. (Euphorbiaceae) illustrate the divergent strategies for the two sexes of wind-pollinated herbs and suggest how they might constrain the breakdown of dioecy (Figure 1a). Mercurialis annua is a complex of European ruderal plants that vary in their sexual systems and inflorescences (Durand 1963; Durand \& Durand 1992; Pannell et al. 2008). Dioecy is ancestral in Mercurialis and is widespread in M. annua in Europe, but monoecy has apparently evolved from dioecy in the Iberian Peninsula and north Africa through the spread of leaky females with an enhanced male function (Obbard et al. 2006). In dioecious populations, males disperse 
produce their flowers on subsessile pedicels in leaf axils (Eppley \& Pannell 2007). The male function of

111 the monoecious $M$. annua is associated with a female phenotype that differs from males in terms of life-

112 history, nitrogen budget and allocation to defense (Hesse \& Pannell 2011b, c; Sanchez-Vilas \& Pannell

113 2011a; Sanchez-Vilas \& Pannell 2011b; Sanchez-Vilas et al. 2011; Labouche \& Pannell 2016; Tonnabel et al. 2017). Importantly, monoecious individuals hold both their male and female flowers in leaf axils, whereas males place flowers on erect inflorescence stalks ('peduncles') (Figure 1a). Pollen dispersed from male peduncles is a $60 \%$ better at siring outcrossed progeny than that from monoecious

117 inflorescences (Eppley \& Pannell 2007). We might view the poor pollen dispersal of monoecious 118 individuals as a 'ghost of dioecy past', in the sense that they are 'haunted' by the legacy of sexual dimorphism inherited from their dioecious ancestors.

Here, we compare the siring success of the typical monoecious individuals with female-like ('F-like') inflorescences with that of hitherto undescribed monoecious individuals of $M$. annua that have longer inflorescences similar to those of males ('M-like'). Populations of the F-like form are widespread around the coast of the Iberian Peninsula, whereas populations of the M-like form are restricted to southern and eastern Spain. Although the two forms rarely co-occur, their distributions are broadly sympatric.

126 The evolutionary paths linking dioecy to the two monoecious forms in M. annua are not well

127 understood. However, pedunculate inflorescences are associated with a Y-linked marker in all lineages 128 that have them, except in M-like monoecious individuals (unpublished data). It is thus likely that 129 peduncles of the M-like lineage are not derived directly from males and have evolved independently.

130 Either way, the peduncle represents a potential improvement on the female-derived monoecious 131 inflorescence. 
competition both with males with the F-like form. We first compared inflorescence morphology and

141 shift from obligate outbreeding to facultative inbreeding, as implied by models of the breakdown of

142 dioecy (Maurice \& Fleming 1995; Wolf \& Takebayashi 2004; Ehlers \& Bataillon 2007; Pannell 2008;

143 Crossman \& Charlesworth 2014).

\section{Materials and Methods}

Study populations, seed collection, and seedling establishment

147 We collected seed from about 30 seed-producing individuals from each of five populations: two

148 androdioecious populations in which males co-occurred at frequencies of approximately $30 \%$ with F-like

149 monoecious individuals (populations $\mathrm{G}$ and $\mathrm{H}$ ); and three monoecious populations with only M-like

151 transplanted seedlings (7 - 10 days after sowing) individually into small pots. When their sex could be

152 determined (at 21 days), we re-transplanted them into $15 \mathrm{~cm}$ diameter pots and established them in

153 their mating arrays outside.

\section{Experimental design}

156 We conducted two experiments to compare male and female components of fitness: in Experiment 1

157 (conducted at Wytham field station, University of Oxford), each monoecious form competed separately 
against males; in Experiment 2 (conducted at the University of Lausanne), we competed the two monoecious forms against one another (Figure 1b). For Experiment 1, we placed males from each androdioecious population with individuals of either the M-like and F-like monoecious forms, yielding

161 ten different common-garden combinations. Each combination was replicated three times (30 mating

162 arrays in total), with seven males and 42 monoecious individuals arranged so that males occurred only once in each row and column. Plants were allowed to mate with one another for four to six weeks, when to establishment of the respective arrays had already dispersed, so that we can be sure that all progeny harvested were sired under the treatment conditions.)

171 established as squares with $25 \mathrm{~F}$-like alternating with $24 \mathrm{M}$-like individuals. Plants were harvested after

172 six weeks.

174 In both experiments, arrays were established across the available area. Arrays for Experiment 1 were

175 tens of meters apart, but for Experiment 2 they had to be placed at approximately three meters from

176 each other. To prevent immigration of pollen from adjacent arrays, we erected plastic barriers $1.5 \mathrm{~m}$ tall

177 between them. A similar setup prevented gene flow among contiguous plots in a previous experiment

178 (Dorken \& Pannell 2009). Any gene flow among arrays would have reduced experimental power, so our 179 results are conservative. 
182 Individuals in the outer edge of all arrays, except males in Experiment 1, were excluded from analysis.

183 Experiment 1 had a final sample size of 851 plants (278 F-like, $373 \mathrm{M}$-like, and 210 male individuals);

184 Experiment 2 had a final sample size of 300 plants (156 F-like and $144 \mathrm{M}$-like individuals). We measured

185 the height of all plants, disregarding the additional height of pedunculate inflorescences. For a

186 subsample of seven plants of each morph and array, we measured the length of five randomly chosen

187 inflorescences (both experiments), and the biomass of all staminate flowers. Previous work has shown

188 that pollen biomass is strongly correlated with the biomass of staminate flowers (Pannell 1997b, c). All

189 plants from both experiments were allowed to dry slowly and release their seeds porous bags. We weighed the seeds of each plant together, as well as the aboveground plant biomass.

\section{Assessment of relative siring success}

193 For Experiment 1, we used the sex ratio in the progeny to estimate the relative siring success of males and monoecious individuals. In M. annua, males are determined by the expression of a dominant allele at a single locus, so that males are heterozygous (i.e., XY) (Russell \& Pannell 2015; Veltsos et al. 2018). Progeny sired by males will thus be $50 \%$ male and $50 \%$ monoecious. Accordingly, we calculated the relative siring success of males in each array as twice the frequency of male progeny, based on 200 progenies grown to sexual maturity per array $(6,000$ individuals in total).

For Experiment 2, we used microsatellites to estimate selfing rates and siring success by F-like- and M-

201 like individuals. We genotyped 30 plants from progeny produced by each of the two monoecious forms

202 from each of the twelve replicate arrays (total of 720 plants), using DNA from young silaca-dried leaves.

203 DNA was extracted with a BioSprint 96 robot (Qiagen), using a BioSprint 96 DNA plant kit (Qiagen).

204 Individuals were scored for five microsatellite markers (Mh14, Mh15, Mh19, Mh52 and Mh91(2) that 205 provide good separation between the monoecious populations sampled (Korbecka et al. 2010). All five 
206

207

208

209

210

211

212

213

214

215

216

217

218

219

220

221

222

223

224

225

226

227

228

markers were amplified in a single multiplexed PCR reaction, following the protocol described in Korbecka et al. (2010). We processed the samples in an ABI 3100 sequencer (Applied Biosystems), and analysed the results with GeneMapper v.4.0 (Applied Biosystems). Individual genotypes were classified as having been sired by a father of the same or a competing phenotype in the array; resolution was insufficient to assign paternity to specific individuals. For populations of the F-like form, we estimated selfing rates using the software RMES (David et al. 2007); those of the M-like form had almost no genetic variability.

\section{Data analysis}

We used mixed models to analyse: plant height; plant biomass; mean length of peduncles per plant; pollen biomass and seed biomass; sex ratio in the progeny of Experiment 1; and, for Experiment 2, the proportion of progeny of parents with different phenotypes. As our primary interest was to determine the functional effect of two contrasting monoecious inflorescence forms in $M$. annua, we defined inflorescence form as a fixed variable, and population within inflorescence form and array as random variables. We tested for a trade-off between male and female reproduction of monoecious individuals in Experiment 1 by fitting a model with pollen mass as the response variable and biomass of seeds, plant biomass and inflorescence form as independent variables, controlling for population and array variation as random terms.

We used Gaussian models for all variables except for the sex ratio and inter-form crossing rate, for which we used a binomial model. Data were log-transformed when necessary to normalize residuals. All data analysis was implemented in R v.3.3.2 (R Development Core Team 2016) using package Ime4 (Bates et al. 2015). Significance of fixed effects and differences between inflorescence forms were evaluated 
using the package ImerTest (Kuznetsova et al. 2017), or z-tests in the case of ratios. Significance of random effects was assessed by likelihood-ratio tests.

\section{Results}

233 Phenotypic variation among inflorescence forms

234 Phenotypic measurements of the three phenotypes (males, F-like- and M-like individuals) were broadly

235 consistent across both Experiments 1 and 2; for brevity, we therefore report measurements for

236 Experiment 1 in the text (Figure 2) and present all data for both experiments in the Supplementary

237 Materials (Tables S1 and S2).

Peduncle length differed between all three phenotypes: peduncles of males were longer than those of

242 like monoecious individuals (Figure $2 \mathrm{~b}$ and Table S1). Males invested much more in male flower

243 production than did both monoecious forms (Figure 2c). Even though there were substantial differences

244 in both vegetative and reproductive traits at the population level (biomass: $X^{2}{ }_{1}=30.6, p<0.001$; seed

245 biomass: $X^{2}{ }_{1}=3.47, p=0.062$; male flower biomass: $X^{2}{ }_{1}=51.2, p<0.001 ;$ Tables S1 and S2), large

246 within-form population variation and low population number rendered differences among forms non-

247 significant (biomass: $t_{1.06}=0.50$, Figure $2 d ; p=0.30$; seed biomass: $t_{16}=1.40$, Figure $2 e ; p=0.18$; pollen

248 biomass: $t_{4}=1.99, p=0.12$, Figure $2 c$ ).

$\underline{\text { Inferred siring success }}$

251 In Experiment 1, in which one or other of the two monoecious forms co-occurred with males, there

252 were substantially more monoecious progeny (as opposed to males) from arrays with M-like (proportion 
$253=0.92+-0.02$ ) than with F-like individuals (proportion $=0.68+ - \pm 0.05 ; z=6.22, p<0.001 ;$ Figure 3a).

254 Given that maleness in M. annua is determined by a dominant allele (see Materials and Methods), we

255 thus inferred that M-like individuals sired 35\% more progeny when competing with males than F-like

256 individuals did. Equivalently, F-like individuals sired 25\% fewer progeny than their F-like counterparts.

257

258 In Experiment 2, in which individuals of the two monoecious forms co-occurred, M-like individuals sired

259 more than three times greater than F-like individuals did $(z=9.3, p<0.001$; Figure $3 b)$. Of progeny with

F-like individuals as both parents, $35 \%$ were self-fertilized. Taking into account progeny of crosses between the two forms, F-like mothers thus self-fertilized a fraction 0.15 of their progeny (Table S3).

262 There was insufficient variation at the microsatellite loci to estimate the selfing rate of M-like

263 individuals. However, if we assume a similar selfing rate for both forms (see Discussion), we may infer

264 that pollen dispersed by F-like individuals in Experiment 2 sired 31\% fewer outcrossed progeny than 265 pollen dispersed by M-like individuals.

266

$\underline{\text { Trade-off between male and female reproduction }}$

268 There was a significant trade-off between male and female allocation to reproduction within

269 populations $\left(t_{4.3}=3.47, p=0.023\right.$; Figure 4$)$, with significant variation in the strength of the trade-off

270 among populations $\left(\mathrm{X}^{2}{ }_{3}=22.2, \mathrm{p}<0.001\right.$; Figure 4). Biomass $\left(\mathrm{t}_{184}=7.04, \mathrm{p}<0.001\right)$ and $\mathrm{M}$-like

271 inflorescence type $\left(t_{10.1}=2.39, p=0.038\right)$ had a positive and significant contribution to the model, i.e.,

272 individuals with higher biomass and peduncles allocated more to male function for a given female

273 allocation.

275 Discussion

276 High siring success of M-like monoecious individuals 
277 Monoecy in M. annua has evolved from dioecy via the modification of females that produce male

278 flowers and that disperse pollen from sub-sessile axillary inflorescences similar to those of females. Our

279 results support the hypothesis that this female-derived inflorescence morphology, a legacy of the

280 breakdown of dioecy, compromises the siring success of monoecious individuals. Monoecious

281 individuals with short female-derived (F-like) inflorescences sired about $25 \%$ fewer progeny in mating

282 arrays with males than a newly characterised form with long, male-like (M-like) inflorescences that

283 occurs in part of the species' range, and they sired 31\% fewer progeny when in direct competition with

284 the M-like form.

285

286 Our results are coherent with those of Eppley and Pannell (2007), who found that pedunculate

287 inflorescences conferred a $60 \%$ siring advantage per pollen grain on males compared with F-like

288 individuals lacking peduncles. Our finding that pollen of the M-like monoecious form has a lower (45\%)

289 siring advantage than the F-like form is consistent with both their shorter peduncles than those of

290 males, and their tendency to produce more pollen and fewer seeds than F-like monoecious individuals.

291 The trade-off between male and female allocations within our arrays indicates that individuals in

292 populations emphasising their male function should have a reduced female function.

294 Our siring estimates assumed that M-like and F-like forms have the same selfing rate. Individuals of the

295 F-like form self-fertilized a proportion 0.15 of their seeds. This relatively low value is consistent with the

296 low selfing rates of F-like monoecious M. annua in both dense experimental and field populations

297 (Eppley \& Pannell 2007; Korbecka et al. 2011). It is also consistent with the presence of males in

298 androdioecious populations of M. annua, because males cannot be maintained with hermaphrodites or

299 monoecious individuals that self-fertilize a large proportion of their progeny (Lloyd 1975b; Charlesworth

300 \& Charlesworth 1978; Charlesworth 1984). M-like individuals should be less likely to fertilize their own 
301

302

303

304

305

306

307

308

309

310

311

312

313

314

315

316

317

318

319

320

321

322

323

324

ovules than F-like individuals, so the selfing rate of the former might be that of the latter. M-like individuals produced more pollen than F-like individuals, and selfing rates individuals in M. annua correlated positively with pollen production in general (unpublished data). Given these opposing effects of the reproductive strategy of $\mathrm{M}$-like individuals, an assumption of similar selfing rates for the two forms seems reasonable.

The higher sex allocation and siring success of M-like monoecious individuals of $M$. annua helps to explain why they rarely co-occur with males (c.f., Pannell et al. 2014), as well as why the two monoecious forms rarely co-occur. Although the weak trade-off among monoecious individuals between their male and female functions might allow co-existence of the two forms (with one emphasising male function and the other emphasising female function), the high fitness of M-like individuals means that Flike individuals should be maintained at low frequencies and could more easily be lost by drift, especially because population size fluctuates so much (Dorken et al. 2017). Similarly, although males produce more pollen and have longer peduncles than M-like individuals, our Experiment 1 showed that the siring success of the latter is sufficient to keep males at low frequency; they, too, might thus easily be lost by drift. The effect of drift and demographic stochasticity has similarly been shown to allow the loss of style morphs of Eichhornia paniculata that are maintained by negative frequency-dependent selection in large populations (Barrett et al. 1989).

The high siring success of the M-like monoecious form of $M$. annua also raises the question of why it has not spread more widely across the Iberian Peninsula, replacing the F-like form. It is possible that the superior mating strategy of the M-like form is costly in ways we have not evaluated, e.g., in terms of physiological and life-history traits that are sexually dimorphic in dioecious or androdioecious $M$. annua (Hesse \& Pannell 2011c; Sanchez-Vilas \& Pannell 2011b; Sanchez-Vilas et al. 2011; Labouche \& Pannell 
2016; Tonnabel et al. 2017). For example, the F-like form might enjoy an advantage over the M-like form during periods of colonization (e.g., because it confers greater reproductive assurance, Friedman \& Barrett 2009a). Such an explanation would be consistent with the metapopulation model proposed by Pannell (1997a), notably if greater seed production by the F-like form allowed it to establish more viable demes early after colonisation (Pannell 2001). Estimates of relative seed production and progeny performance from a wider sample of populations would help to evaluate this possibility.

\section{Sexual dimorphism and the stabilization of dioecy}

Variation in inflorescence morphology in $M$. annua illustrates how sexual dimorphism might stabilize the maintenance of separate sexes in dioecious plants. Sex-allocation theory predicts that if either the male or the female sexual function (or both functions together) have accelerating fitness gain curves, then the 'fitness set' is concave, and dioecy should be evolutionarily stable (Charnov et al. 1976; Charnov 1982; West 2009). Sexual dimorphism likely allows unisexuals to perform better than hermaphrodites in their corresponding sexual function, which should enhance the concavity of the fitness set (Figure 5). Due to lack of specialised structures for pollen dispersal, the male fitness gain curve of F-like hermaphrodites in M. annua is probably saturating. In contrast, our results suggest that the superior inflorescence structure of the M-like morph likely diminish this saturating tendency. (Saturation of female fitness gain curves likely remain unchanged.) If so, we suggest that the fitness set of the M-like form should relax conditions for their invasion into a dioecious population and a transition to functional hermaphroditism, particularly under intense competition for outcross siring (Pannell 2001).

Figure 6 sets out a path that might commonly be followed when dioecy breaks down. In recently evolved dioecious populations, the fitness of males and females will be compromised by the expression of genes in individuals of one sex that are better suited to performance of the other, or genes 
underpinning a reproductive, physiological, life-history, or defence strategy that is not optimised for its

own sex. Over time, selection should act to reduce trait correlations between males and females (Lande 1980), optimising male and female phenotypes differently in a sexually dimorphic population (Figure 6a). It is also possible that selection for sexual specialisation sometimes coincides with the transition

353 from hermaphroditism to dioecy (Willson 1979; Bawa 1980; Givnish 1982) - although the hypothesis is controversial (Thomson \& Barrett 1981; Charlesworth 1985). Either way, males and females of dioecious populations end up with phenotypes that enhance their own sex function, but that might not do so for the other, and might indeed be deleterious (Connallon \& Clark 2014). et al. 2017; Kafer et al. 2017) presumably begin with selection of males or females with a leaky sexual function, and the derived hermaphrodites (or monoecious individuals) should inherit the secondary sexual phenotype associated with their ancestral sexual function, male or female (Figure 6b). Our results suggest that this ancestral phenotype is antagonistic to the newly acquired function in monoecious $M$. annua, and similar reasoning may hold for transitions from dioecy to hermaphroditism more generally. For example, many dioecious lineages may revert to hermaphroditism via gynodioecy through the selection of leaky males, which are much more common than leaky females (Ehlers \& Bataillon 2007; Cossard et al. 2018). In these lineages, hermaphrodites derived from leaky males will tend to express their acquired female function in the context of floral, physiological, life-history or defense traits that have been selected to optimize siring success, not seed or fruit production. With time, we expect selection to mold the hermaphroditic phenotypes in ways that overcome this ghost of dioecy, finding solutions that optimize both male and female functions and, for example, avoiding their interference 371 (Figure 6c). 
373 The above reasoning implies that sexual dimorphism should constrain the breakdown of dioecy when

374 outcrossing is favored by establishing populations with a concave fitness set that become more resistant

375 to the invasion of hermaphrodites. Indeed, once sexual dimorphism has evolved, it is difficult to imagine

376 what could allow a transition from dioecy to hermaphroditism without an accompanying change in the

377 mating system. In the case of $M$. annua, monoecy likely evolved from dioecy as a selfing mechanism in

378 response to selection for reproductive assurance in sparse populations (Hesse \& Pannell 2011a;

379 Labouche et al. 2015), during episodes of range expansion (Pujol \& Pannell 2008; Pujol et al. 2009), or

380 during colonization of disturbed habitat in metapopulations (Pannell 2001; Pannell \& Dorken 2006;

381 Pannell et al. 2008), and mate limitation has been invoked for the breakdown of dioecy in other plant

382 and animal lineages (Baker \& Cox 1984; Charlesworth 1993; Maurice \& Fleming 1995; Pannell 2002;

383 Wolf \& Takebayashi 2004; Ehlers \& Bataillon 2007; Crossman \& Charlesworth 2014). Our study

384 emphasizes that such advantages must overcome the sometimes substantial benefits of sexual

385 specialization enjoyed by males and females. Thus, while reproductive assurance may favor transitions

386 from dioecy to hermaphroditism, sexual dimorphism (a legacy of selection under dioecy), should resist

387 such transitions in outcrossing populations. We suggest that dioecy is thus most likely to break down

388 when accompanied by a shift from outcrossing to selfing or mixed mating.

Acknowledgements

391 We thank Nicolas Ruch for help with the array experiments, Laure Olazcuaga for help in the laboratory

392 and field, Crispin Jordan, Paris Veltsos, Wen-Juan Ma, Marcos Méndez Iglesias, and two anonymous

393 referees for comments on the manuscript, and the Swiss National Science Foundation for funding to JRP.

References 
Aylor, D.E., Schultes, N.P. \& Shields, E.J. (2003). An aerobiological framework for assessing crosspollination in maize. Agric. For. Meteorol., 119, 111-129.

Baker, H.G. \& Cox, P.A. (1984). Further thoughts on dioecism and islands. Ann. Mo. Bot. Gard., 71, 244253.

Barrett, S.C.H. \& Hough, J. (2013). Sexual dimorphism in flowering plants. J. Exp. Bot., 64, 67-82.

Barrett, S.C.H., Morgan, M.T. \& Husband, B.C. (1989). The dissolution of a complex genetic polymorphism: the evolution of self-fertilization in tristylous Eichhornia paniculata (Pontederiaceae). Evolution, 43, 1398-1416.

Bates, D., Maechler, M., Bolker, B. \& Walker, S. (2015). Fitting linear mixed-effects models using Ime4. J. Stat. Soft., 67, 1-48.

Bawa, K.S. (1980). Evolution of dioecy in flowering plants. Annu. Rev. Ecol. Syst., 11, 15-39.

Becerra, J.X. \& Venable, D.L. (1999). Nuclear ribosomal DNA phylogeny and its implications for evolutionary trends in Mexican Bursera (Burseraceae). Amer. J. Bot., 86, 1047-1057.

Bull, J.J. \& Charnov, E.L. (1985). On irreversible evolution. Evolution, 39, 1149-1155.

Charlesworth, D. (1984). Androdioecy and the evolution of dioecy. Biol. J. Linn. Soc., 23, 333-348.

Charlesworth, D. (1985). Distribution of dioecy and self-compatibility in angiosperms. In: Evolution: Essays in honour of John Maynard Smith (eds. Harvey, PH, Slatkin, M \& Greenwood, PJ). Cambridge University Press Cambridge, pp. 237-268.

Charlesworth, D. (1993). Save the male. Curr. Biol., 3, 155-157.

Charlesworth, D. \& Charlesworth, B. (1978). A model for the evolution of dioecy and gynodioecy. Amer. Nat., 112, 975-997.

Charnov, E.L. (1982). The Theory of Sex Allocation. Princeton University Press, Princeton, NJ.

Charnov, E.L., Maynard Smith, J. \& Bull, J.J. (1976). Why be an hermaphrodite? Nature, 263, 125-126. 
Connallon, T. \& Clark, A.G. (2014). Evolutionary inevitability of sexual antagonism. Proc. Roy. Soc. Lond.

$$
\text { B., } 281,7 .
$$

Cossard, G., Tudor, E. \& Pannell, J.R. (2018). Functional components of sex inconstancy in the dioecious coloniser Mercurialis annua. Amer. J. Bot., (resubmitted, pending minor revision).

Cox, R.M. \& Calsbeek, R. (2009). Sexually antagonistic selection, sexual dimorphism, and the resolution of intralocus sexual conflict. Amer. Nat., 173, 176-187.

Cresswell, J.E., Krick, J., Patrick, M.A. \& Lahoubi, M. (2010). The aerodynamics and efficiency of wind pollination in grasses. Func. Ecol., 24, 706-713.

Crossman, A. \& Charlesworth, D. (2014). Breakdown of dioecy: models where males acquire cosexual functions. Evolution, 68, 426-440.

Darwin, C. (1871). The Descent of Man, and Selection in Relation to Sex. John Murray, London.

David, P., Pujol, B., Viard, F., Castella, V. \& Goudet, J. (2007). Reliable selfing rate estimates from imperfect population genetic data. Mol. Ecol., 16, 2474-2487.

Dorken, M.E., Freckleton, R.P. \& Pannell, J.R. (2017). Small-scale and regional spatial dynamics of an annual plant with contrasting sexual systems. J. Ecol., 105, 1044-1057.

Dorken, M.E. \& Pannell, J.R. (2009). Hermaphroditic sex allocation evolves when mating opportunities change. Curr. Biol., 19, 514-517.

Durand, B. (1963). Le complèxe Mercurialis annua L. s.I.: une étude biosystématique. Ann. Sci. Nat. Bot. Paris, 12, 579-736.

Durand, R. \& Durand, B. (1992). Dioecy, monoecy, polyploidy and speciation in the annual Mercuries. Bull. Soc. Bot. France Lett. Bot., 139, 377-399.

Ehlers, B.K. \& Bataillon, T. (2007). 'Inconstant males' and the maintenance of labile sex expression in subdioecious plants. New Phytol., 174, 194-211. 
442 443

444

445

446

447

448

449

450

451

452

453

454

455

456

457

458

459

460

461

462

463

464

Eppley, S.M. \& Pannell, J.R. (2007). Density-dependent self-fertilization and male versus hermaphrodite siring success in an androdioecious plant. Evolution, 61, 2349-2359.

Fairbairn, D.J., Blanckenhorn, W.U. \& Székely, T. (2007). Sex, size and gender roles. Evolutionary studies of sexual size dimorphism. Oxford University Press, Oxford.

Friedman, J. \& Barrett, S.C.H. (2008). A phylogenetic analysis of the correlates and evolution of wind pollination in the angiosperms. Int. J. PI. Sc., 169, 49-58.

Friedman, J. \& Barrett, S.C.H. (2009a). The consequences of monoecy and protogyny for mating in windpollinated Carex. New Phytol., 181, 489-497.

Friedman, J. \& Barrett, S.C.H. (2009b). Wind of change: new insights on the ecology and evolution of pollination and mating in wind-pollinated plants. Ann. Bot., 103, 1515-1527.

Galonka, A.M., Sakai, A.K. \& Weller, S.G. (2005). Wind pollination, sexual dimorphism, and changes in floral traits of Schiedea (Caryophyllaceae). Amer. J. Bot., 92, 1492-1502.

Geber, M.A., Dawson, T.E. \& Delph, L.F. (1999). Gender and Sexual Dimorphism in Flowering Plants. Springer Heidelberg.

Givnish, T.J. (1982). Outcrossing versus ecological constraints in the evolution of dioecy. Amer. Nat., 119, 849-865.

Goldberg, E.E., Otto, S.P., Vamosi, J.C., Mayrose, I., Sabath, N., Ming, R. et al. (2017). Macroevolutionary synthesis of flowering plant sexual systems. Evolution, 71, 898-912.

Harder, L.D. \& Prusinkiewicz, P. (2013). The interplay between inflorescence development and function as the crucible of architectural diversity. Ann. Bot., 112, 1477-1493.

Harrington, M.G. \& Gadek, P.A. (2010). Phylogenetics of hopbushes and pepperflowers (Dodonaea, Diplopeltis-Sapindaceae), based on nuclear ribosomal ITS and partial ETS sequences incorporating secondary-structure models. Aust. Syst. Bot., 23, 431-442. 
Harris, M.S. \& Pannell, J.R. (2010). Canopy seed storage is associated with sexual dimorphism in the woody dioecious genus Leucadendron. J. Ecol., 98, 509-515.

Heilbuth, J.C. (2000). Lower species richness in dioecious clades. Amer. Nat., 156, 221-241.

Hesse, E. \& Pannell, J.R. (2011a). Density-dependent pollen limitation and reproductive assurance in a wind-pollinated herb with contrasting sexual systems J. Ecol., 99, 1531-1539.

Hesse, E. \& Pannell, J.R. (2011b). Sexual dimorphism in a dioecious population of the wind-pollinated herb Mercurialis annua: the interactive effects of resource availability and competition. Ann. Bot., 107, 1039-1045.

Hesse, E. \& Pannell, J.R. (2011c). Sexual dimorphism in androdioecious Mercurialis annua, a windpollinated herb. Int. J. PI. Sc., 172, 49-59.

Kafer, J., de Boer, H.J., Mousset, S., Kool, A., Dufay, M. \& Marais, G.A.B. (2014). Dioecy is associated with higher diversification rates in flowering plants. J. Evol. Biol., 27, 1478-1490.

Kafer, J., Marais, G.A.B. \& Pannell, J.R. (2017). On the rarity of dioecy in flowering plants. Mol. Ecol., 26, $1225-1241$.

Käfer, J. \& Mousset, M. (2014). Standard sister clade comparison fails when testing derived character states. Syst. Biol., 63, 601-609.

Korbecka, G., Hamilton, A. \& Pannell, J.R. (2011). Mixed mating in polyploid androdioecious populations of Mercurialis annua estimated using multilocus genotypes in progeny arrays. Ann. Bot., 107, 1057-1061.

Korbecka, G., Rymer, P.D., Harris, S.A. \& Pannell, J.R. (2010). Solving the problem of ambiguous paralogy for marker loci: microsatellite markers with diploid inheritance in allohexaploid Mercurialis annua (Euphorbiaceae). J. Hered., 101, 504-511. 
487 488

489

490

491

492

493

494

495

496

497

498

499

500

501

502

503

504

505

506

507

508

509

Krahenbuhl, M., Yuan, Y.M. \& Kupfer, P. (2002). Chromosome and breeding system evolution of the genus Mercurialis (Euphorbiaceae): implications of ITS molecular phylogeny. PI. Syst. Evol., 234, $155-170$.

Kuznetsova, A., Brockhoff, P.B. \& Christensen, R.H.B. (2017). ImerTest Package: Tests in Linear Mixed Effects Models. J. Stat. Soft., 82, 1-26.

Labouche, A.M. \& Pannell, J.R. (2016). A test of the size-constraint hypothesis for a limit to sexual dimorphism in plants. Oecologia, 105, 197-208.

Labouche, A.M., Richards, S.A. \& Pannell, J.R. (2017). Effects of pollination intensity on offspring number and quality in a wind-pollinated herb. J. Ecol., 105, 197-208.

Lande, R. (1980). Sexual dimorphism, sexual selection and adaptation in polygenic characters. Evolution, 34, 292-305.

Lloyd, D.G. (1975a). Breeding systems in Cotula IV. Reversion from dioecy to monoecy. New Phytol., 74, 125-145.

Lloyd, D.G. (1975b). The maintenance of gynodioecy and androdioecy in angiosperms. Genetica, 45, 325339.

Lloyd, D.G. \& Webb, C.J. (1977). Secondary sex characters in plants. Bot. Rev., 43, 177-216.

Maurice, S. \& Fleming, T.H. (1995). The effect of pollen limitation on plant reproductive systems and the maintenance of sexual polymorphisms. Oikos, 74, 55-60.

Moore, J.C. \& Pannell, J.R. (2011). Sexual selection in plants. Curr. Biol., 21, R176-R182.

Obbard, D.J., Harris, S.A., Buggs, R.J.A. \& Pannell, J.R. (2006). Hybridization, polyploidy, and the evolution of sexual systems in Mercurialis (Euphorbiaceae). Evolution, 60, 1801-1815.

Pannell, J. (1997a). The maintenance of gynodioecy and androdioecy in a metapopulation. Evolution, 51, 10-20. 
Pannell, J. (1997b). Variation in sex ratios and sex allocation in androdioecious Mercurialis annua. J.

$$
\text { Ecol., 85, 57-69. }
$$

Pannell, J. (1997c). Widespread functional androdioecy in Mercurialis annua L. (Euphorbiaceae). Biol. J. Linn. Soc., 61, 95-116.

Pannell, J.R. (2001). A hypothesis for the evolution of androdioecy: the joint influence of reproductive assurance and local mate competition in a metapopulation. Evol. Ecol., 14, 195-211.

Pannell, J.R. (2002). The evolution and maintenance of androdioecy. Annu. Rev. Ecol. Syst., 33, 397-425.

Pannell, J.R. (2008). Consequences of inbreeding depression due to sex-linked loci for the maintenance of males and outcrossing in branchiopod crustaceans. Genet. Res., 90, 73-84.

Pannell, J.R. \& Dorken, M.E. (2006). Colonisation as a common denominator in plant metapopulations and range expansions: effects on genetic diversity and sexual systems. Landscape Ecol., 21, 837848.

Pannell, J.R., Dorken, M.E., Pujol, B. \& Berjano, R. (2008). Gender variation and transitions between sexual systems in Mercurialis annua (Euphorbiaceae). Int. J. PI. Sc., 169, 129-139.

Pannell, J.R., Eppley, S.M., Dorken, M.E. \& Berjano, R. (2014). Regional variation in sex ratios and sex allocation in androdioecious Mercurialis annua. J. Evol. Biol., 27, 1467-1477.

Pujol, B. \& Pannell, J.R. (2008). Reduced responses to selection after species range expansion. Science, $321,96$.

Pujol, B., Zhou, S.R., Sahchez-Vilas, J. \& Pannell, J.R. (2009). Reduced inbreeding depression after species range expansion. Prod. Nat. Acad. Sci. USA., 106, 15379-15383.

R Development Core Team, X. (2016). R: A Language and Environment for Statistical Computing. R Foundation for Statistical Computing Vienna, Austria.

Renner, S.S. (2014). The relative and absolute frequencies of angiosperm sexual systems: dioecy, monoecy, gynodioecy, and an updated online database. Amer. J. Bot., 101, 1588-1596. 
Renner, S.S. \& Ricklefs, R.E. (1995). Dioecy and its correlates in the flowering plants. Amer. J. Bot., 82, 596-606.

Russell, J.R.W. \& Pannell, J.R. (2015). Sex determination in dioecious Mercurialis annua and its close diploid and polyploid relatives. Heredity, 114, 262-271.

Sabath, N., Goldberg, E.E., Glick, L., Einhorn, N., Ashman, T.L., Ming, R. et al. (2016). Dioecy does not consistently accelerate or slow lineage diversification across multiple genera of angiosperms. New Phytol., 209, 1290-1300.

Sanchez-Vilas, J. \& Pannell, J. (2011a). Sex-differential herbivory in androdioecious Mercurialis annua. PLoS One, e22083.

Sanchez-Vilas, J. \& Pannell, J.R. (2011b). Sexual dimorphism in resource acquisition and deployment: both size and timing matter. Ann. Bot., 107, 119-126.

Sanchez-Vilas, J., Turner, A. \& Pannell, J.R. (2011). Sexual dimorphism in intra- and interspecific competitive ability of the dioecious herb Mercurialis annua. PI. Biol., 13, 218-222.

Schaefer, H. \& Renner, S.S. (2010). A three-genome phylogeny of Momordica (Cucurbitaceae) suggests seven returns from dioecy to monoecy and recent long-distance dispersal to Asia. Mol. Phylogenet. Evol., 54, 553-560.

Soza, V.L. \& Olmstead, R.G. (2010). Evolution of breeding systems and fruits in New World Galium and relatives (Rubiaceae). Amer. J. Bot., 97, 1630-1646.

Sweeney, P.W. (2008). Phylogeny and floral diversity in the genus Garcinia (Clusiaceae) and relatives. Int. J. PI. Sc., 169, 1288-1303.

Thomson, J.D. \& Barrett, S.C.H. (1981). Selection for outcrossing, sexual selection, and the evolution of dioecy in plants. Amer. Nat., 118, 443-449.

Tonnabel, J., David, P. \& Pannell, J.R. (2017). Sex-specific strategies of resource allocation in response to competition for light in a dioecious plant. Oecologia, 185, 675-686. 

Amer. Nat., 163, 122-137. 281.
Urzay, J., Smith, S.G.L., Thompson, E. \& Glover, B.J. (2009). Wind gusts and plant aeroelasticity effects on the aerodynamics of pollen shedding: A hypothetical turbulence-initiated wind-pollination mechanism. J. Theor. Biol., 259, 785-792.

Veltsos, P., Cossard, G., Beaudoing, E., Genséric, B., Roux, C., Gonzalez-Martinez, S.C. et al. (2018). The size and content of the sex-determining region of the $\mathrm{Y}$ chromosome in dioecious Mercurialis annua, a plant with homomorphic sex chromosomes. Genes, 9, 277.

Volz, S.M. \& Renner, S.S. (2008). Hybridization, polyploidy and evolutionary transitions between monoecy and dioecy in Bryonia (Cucurbitaceae). Amer. J. Bot., 95, 1297-1306.

Weberling, F. (1992). Morphology of flowers and inflorescences. Cambridge University Press, Cambridge. Weiblen, G.D., Oyama, R.K. \& Donoghue, M.J. (2000). Phylogenetic analysis of dioecy in monocotyledons. Amer. Nat., 155, 46-58.

West, S.A. (2009). Sex Allocation. Princeton University Press, Princeton.

Westergaard, M. (1958). The mechanism of sex determination in dioecious plants. Adv. Genet., 9, 217-

Whitehead, D.R. (1983). Wind pollination: some ecological and evolutionary perspectives. In: Pollination Biology (ed. Real, L). Academic Press Orlando, Florida, pp. 97-109.

Willson, M.F. (1979). Sexual selection in plants. Amer. Nat., 113, 777-790.

Wolf, D.E. \& Takebayashi, N. (2004). Pollen limitation and the evolution of androdioecy from dioecy. 
Figure 1. (a) Cartoon drawings illustrating the inflorescence variation in Mercurialis annua between

579 females, F-like monoecious individuals, M-like monoecious individuals, and males. Black circles represent female flowers or fruits. White circles represent male flowers. (b) Experimental design for Experiments 1 and 2. There were three replicates for each combination of competing populations

582 indicated. In Experiment 1, males from each of two androdioecious populations competed either against female-like monoecious individuals from each of the same two populations, or male-like monoecious individuals from each of three populations. In Experiment 2, female-like monoecious individuals from each of two of their populations competed against male-like monoecious individuals from each of two of their populations.

Figure 2. Mean values for several size and allocation traits for males, female-like (F-like) and male-like individuals (M-like) of Mercurialis annua: (a) height including inflorescence; (b) dry biomass; (c) male flower biomass; (d) total biomass of seeds produced; (e) peduncle length. Measurements were taken from mating arrays in Experiment 1 under uniform environmental conditions. Error bars show the standard error. Significant pairwise differences $(p<0.05)$ are indicated by different low case letters. Sample sizes for panels (b), (d) and (e): males $=210, F-l i k e=278, M$-like $=373$. Sample sizes for panels (a) and $(\mathrm{c})$ : males $=210$, F-like $=84$, M-like $=126$.

Figure 3. (a) Mean proportion of males in the progeny of female-like (F-like) and male-like (M-like)

597 hermaphrodites grown in mating arrays together with males (Experiment 1). (b) Proportion of progeny of female-like and male-like individuals attributable to crosses with the other morph after mating in mixed mating arrays in Experiment 2. Significant pairwise differences $(\mathrm{p}<0.05)$ are indicated by different low case letters. Samples sizes: (a) M-like = 3600, F-like = 2400; (b) M-like = 360, F-like = 360. 
602 Figure 4. Trade-off between male and female allocation at the individual plant and population

603 level from Experiment 1. (a) Male vs. female average relative reproductive allocation at the population

604 level. Hollow circles represent female-like populations; solid circles represent male-like populations. (b),

605 (d) and (f) male and female relative reproductive allocation at the individual level in each of three

606 populations with male-like individuals (populations A, C and D, respectively). (c) and (e) male and female

607 relative reproductive allocation at the individual level in two female-like populations (populations $\mathrm{G}$ and

$608 \mathrm{H}$, respectively). Relative reproductive allocation was estimated as the biomass allocated to either sex

609 divided by total plant biomass.

611 Figure 5. Heuristic schema illustrating the hypothesized influence of the adoption of a specialized male-

612 like inflorescence by males or hermaphrodites on the fitness set relating male to female components of

613 fitness. The diagonal straight line depicts a linear fitness set representing a simple tradeoff between

614 male and female functions of a wind-pollinated plant like Mercurialis annua, in the absence of any

615 sexual specialization by males or females. Females (open white circle) occupy the extreme female end of

616 this line. Female-like individuals allocating resources to pollen would occupy positions along the straight-

617 line diagonal. Adoption of a specialized male inflorescence increases the male component of fitness

618 (closed black circle), so that males have a fitness greater than that achievable simply by allocating all

619 reproductive resources to male function (represented by the interval A). The adoption by males of the

620 specialized inflorescence effectively renders the fitness set concave (dashed curve). Adoption by

621 hermaphrodites of a specialized male-like inflorescence increases their male fitness component above

622 that achievable by female-like hermaphrodites (interval B), effectively removing the concavity of the

623 fitness set (dotted line). In our experiments, intervals A and B increased male fitness by about $60 \%$ and

$62445 \%$ above the straight-line diagonal. See text for details. 
626 Figure 6. General evolutionary path envisaged for the breakdown of dioecy in flowering plants. (a) Over

627 the course of its persistence, a dioecious population evolves secondary sexual dimorphism, with males

628 and females expressing phenotypes that are optimized for their respective sexual functions. (b) Dioecy

629 breaks down in response to selection for reproductive assurance and a capacity to self-fertilize by

630 individuals with leaky sex expression. The hermaphrodites thus formed must express their newly

631 acquired sex function in the context of a phenotype optimized for the other sex function. This

632 compromised phenotype is what we refer to as the 'ghost of dioecy'. (c) With time, selection for

633 improved reproductive performance through both sexual functions molds the hermaphroditic strategy,

634 finding phenotypes that improve one sexual function without, or with diminished, deleterious effects to

635 the other.

636 
Figure 1.

(a)

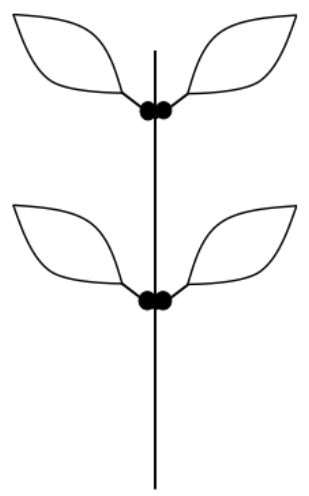

Female

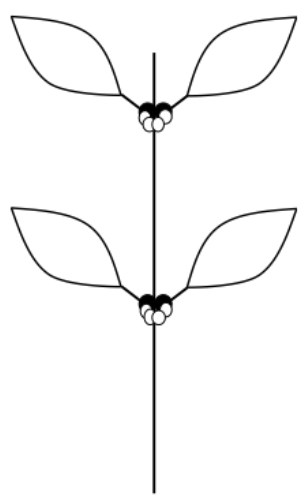

F-like

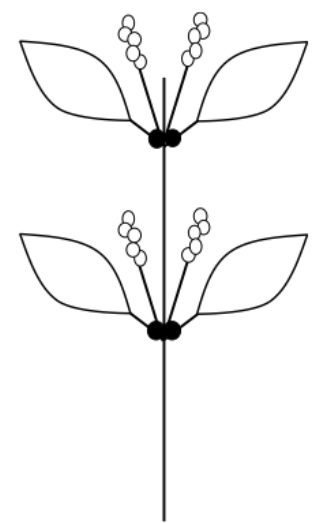

M-like

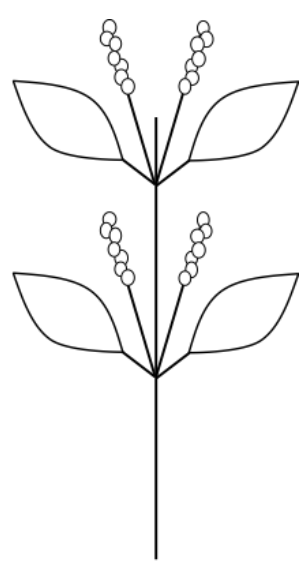

Male

Monoecious

(b)

Experiment 1

Monoecious morphs

Males $\left\{\begin{array}{c|c|c|c|c|c|}\hline & \text { Pop G } & \text { Pop H } & \text { Pop A } & \text { Pop C } & \text { Pop D } \\ \hline \text { Pop G } & 3 & 3 & 3 & 3 & 3 \\ \hline \text { Pop H } & 3 & 3 & 3 & 3 & 3 \\ \hline\end{array}\right.$

Experiment 2

M-like

F-like $\left\{\begin{array}{c|c|c|}\right.$\cline { 2 - 3 } & \text {$Pop A } & \text { Pop D } \\ \hline \text { Pop G } & 3 & 3 \\ \hline \text { Pop H } & 3 & 3 \\ \hline\end{array}$


a)

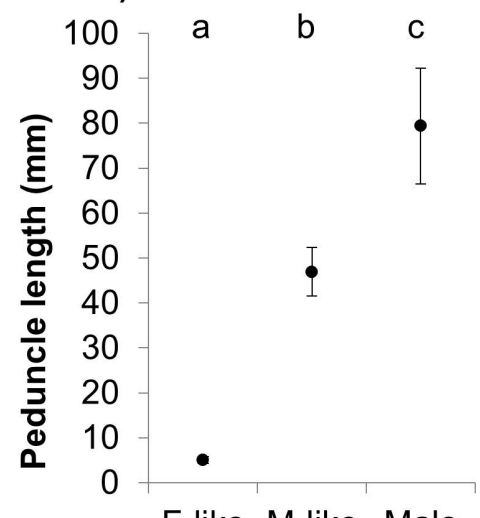

c)

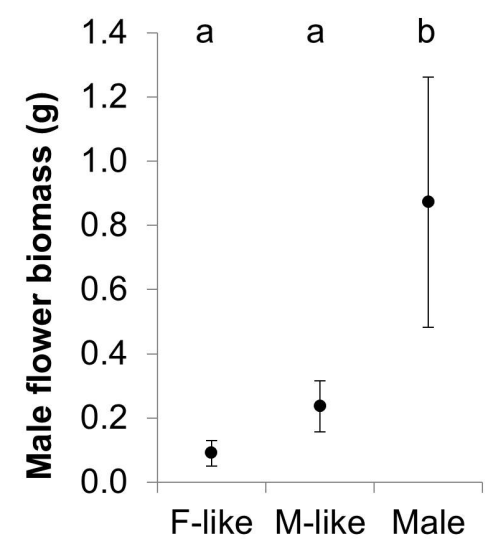

e)

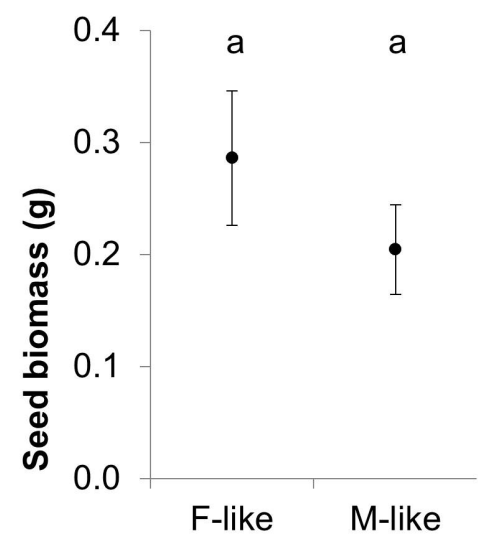

b)

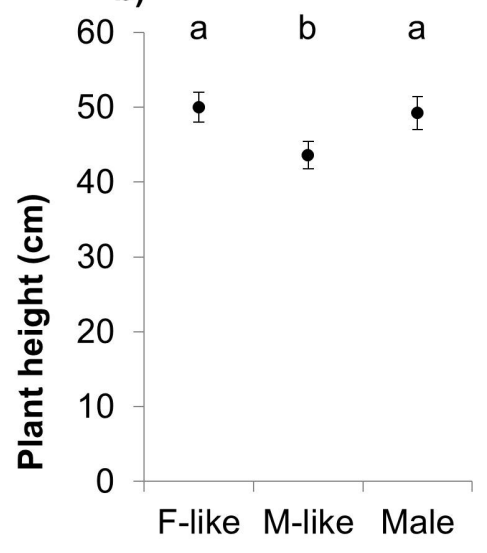

d)

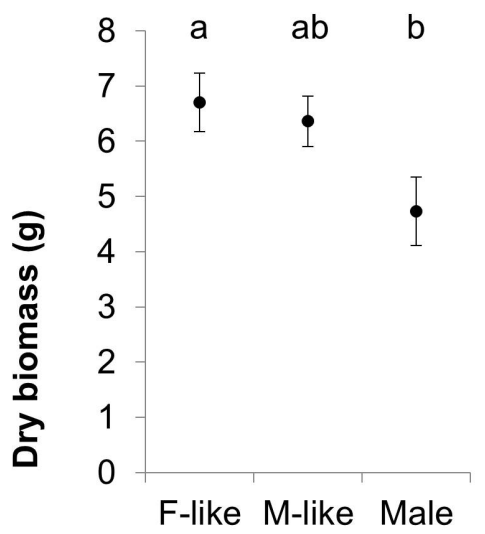


641 Figure 3.

a)

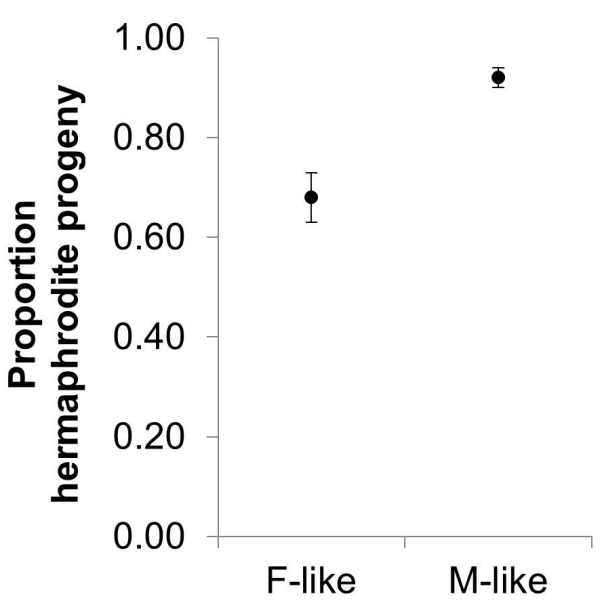

b)

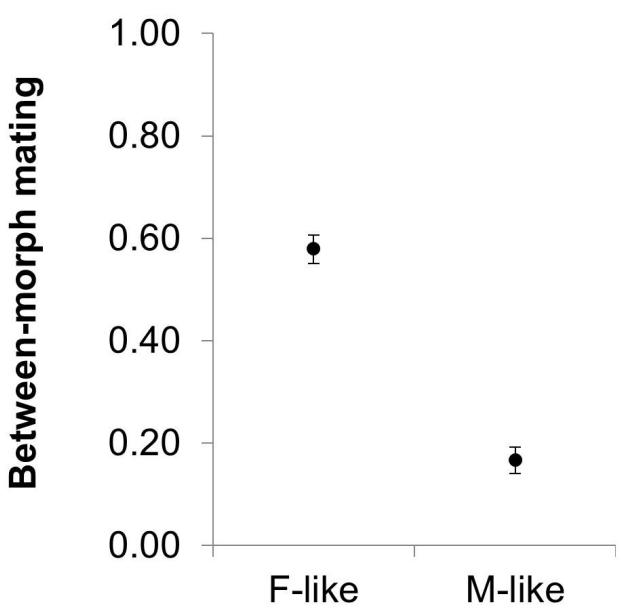


644 Figure 4.

645

a)

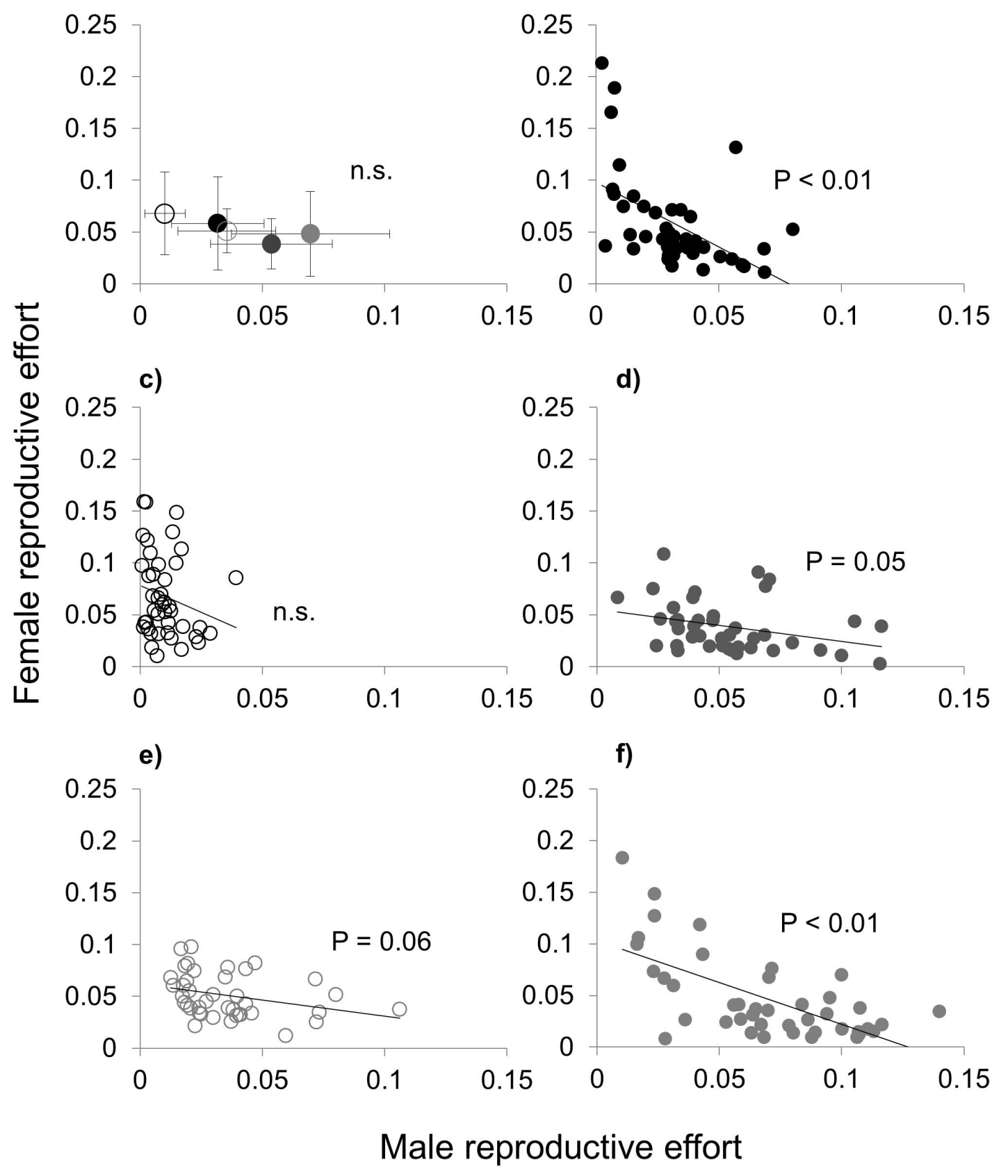


$648 \quad$ Figure 5.

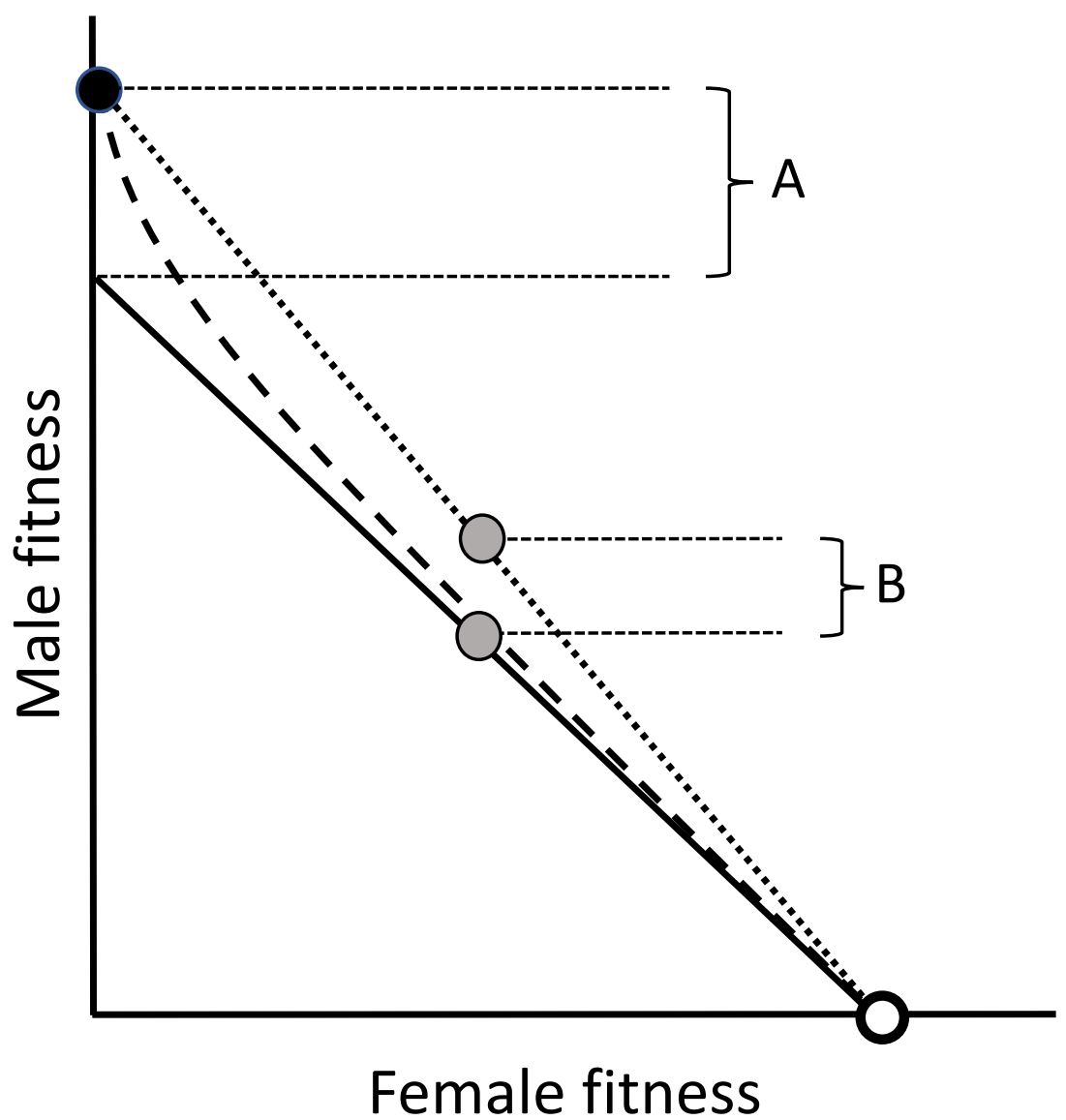

649

650 
651 Figure 6.

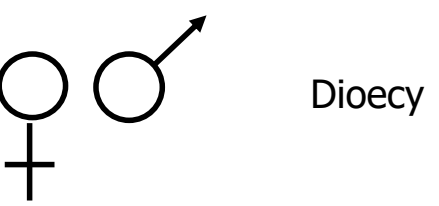

(a)

- Selection for sexual specialization

- Resolution of sexual antagonism by sexual dimorphism (SD)

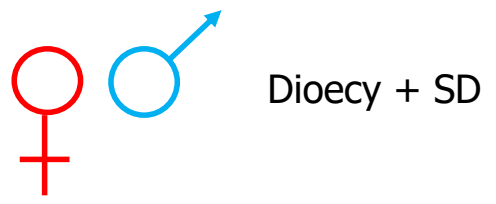

(b)

- $\quad$ Selection for capacity to self-fertilize

- Male function expressed in a compromised female background (the 'ghost of dioecy')

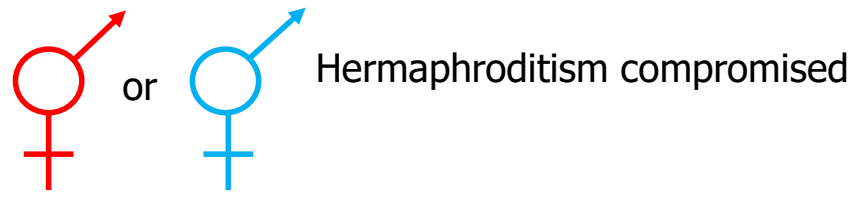

